\title{
L'agricoltura contadina fra istanze locali e globali
}

\section{Introduzione}

Nell'esprimere in più occasioni che la scienza del diritto agrario deve essere capace di affrontare le sfide contemporanee (come la food security, la food safety e la tutela del clima) in una prospettiva sia locale che regionale (a livello di Unione europea) e internazionale, Roman Budzinowski invita i giusagraristi a valutare l'adeguatezza degli strumenti giuridici esistenti a vario livello rispetto a tali sfide e l'opportunità di proporre nuove soluzioni facendo tesoro dell'evoluzione delle ricerche scientifiche ${ }^{1}$.

In quest'ottica, appare interessante esaminare il tema dell'agricoltura cosiddetta contadina in quanto attualmente è oggetto di un disegno di legge in discussione al Parlamento italiano, che si è riuscito a imporre all'attenzione politica anche grazie al clima favorevole che si è creato in seguito all'approvazione, nel 2018, della Dichiarazione dell'ONU sui diritti dei contadini e delle altre persone che lavorano nelle aree rurali. Il dibattito intorno alla nozione di agricoltura contadina e alle istanze di tutela dei contadini offre, infatti, interessanti spunti di riflessione sul ruolo dell'agricoltura non

* Consiglio Nazionale delle Ricerche, sez. DAIC-ISGI, Italia.

${ }^{1} \mathrm{~V}$., in tal senso, R. Budzinowski, Il diritto agrario di fronte alle sfide contemporanee. Alcune riflessioni, "Agricoltura Istituzioni Mercati” 2013, n. 1-2, p. 145 ss.; idem, Contemporary challenges of agricultural law: among globalization, regionalization and locality (introductory considerations), "Przegląd Prawa Rolnego" 2018, n. 1, p. 13 ss. e spec. p. 23. Sul diritto agrario come diritto "in movimento", v. anche idem, Considerazioni sullo sviluppo del diritto agrario (Riflessioni intorno ai diritti della terra e del mercato agroalimentare), in: I diritti della terra e del mercato agroalimentare - Liber amicorum Alberto Germanò, t. II, Milano 2016, p. 1145 ss. 
industrializzata e di piccole dimensioni nel perseguire obiettivi di interesse globale come la food security e la lotta al cambiamento climatico e, più in generale, la sfida dello sviluppo sostenibile posta dall'ONU con l'Agenda $2030^{2}$, nonché sugli istituti tradizionali del diritto agrario italiano, quali quello dell'imprenditore agricolo e del coltivatore diretto.

\section{L'“agricoltura contadina": dalle istanze della società civile al dibattito parlamentare italiano}

L'approdo nel Parlamento italiano di un disegno di legge sulla tutela e sulla valorizzazione dell'agricoltura contadina è il frutto di un movimento nato dal basso che si oppone, da un lato, al modello dell'agricoltura meccanizzata e industrializzata oggi dominante come simbolo di progresso e di modernizzazione e, dall'altro, alla concezione dei contadini e del mondo contadino come residuo del passato, destinato ineluttabilmente a scomparire o tutt'al più relegato a "decorazione del paesaggio e folklore"3.

La Campagna popolare per lanciare una proposta normativa a livello statale è formalmente partita nel 2009 e mira ad ottenere il "riconoscimento sociale ed economico del ruolo indispensabile del lavoro nei campi per la produzione di cibo e del suo agire collettivo; del lavoro agricolo fondamentale per il mantenimento di comunità rurali attive, attrattive e diversificate; del valore culturale dell'attività agricola e della sua capacità di prevenire la desertificazione sociale e l'esodo verso le aree urbane"4. In sostanza, la Campagna è diretta al sostegno di un tipo di agricoltura basato sulle piccole e piccolissime imprese non meccanizzate né innovative che, pur essendo il più diffuso in $\mathrm{Italia}^{5}$, si

${ }^{2}$ Risoluzione adottata dall'Assemblea Generale il 25 settembre 2015 "Trasformare il nostro mondo: 1'Agenda 2030 per lo Sviluppo Sostenibile", reperibile dal sito https://www.un.org/ sustainabledevelopment/development-agenda/. Sugli obiettivi dell'Agenda 2030 che promuovono l'agricoltura sostenibile, v., per tutti, E. Cristiani, Quali regole per un'agricoltura sostenibile, "Rivista di diritto agrario" 2019, n. 1, p. 645 ss.

${ }^{3}$ V., in tal senso, A. Onorati, Agricoltura italiana e agricoltura contadina. La necessità di un quadro giuridico specifico, "Parole chiave" 2017, n. 2, p. 94, da cui sono tratte le parole tra virgolette, ispirate da uno dei documenti della Campagna a sostegno di una legge quadro per il settore, che è stato pubblicato in calce al suo articolo. Sulla Campagna popolare per l'agricoltura contadina, v. https://www.agricolturacontadina.org/.

${ }^{4}$ A. Onorati, Agricoltura italiana..., p. 95. L'A. è membro della Campagna Popolare per l'Agricoltura Contadina ed è stato per molti anni Presidente dell'Ong Centro Internazionale Crocevia (https://www.croceviaterra.it/).

${ }^{5}$ L'Istituto Nazionale di Statistica-ISTAT, nel report del 2 dicembre 2019 relativo alla struttura e alle caratteristiche delle unità economiche del settore agricolo nel 2017, rileva che "Le unità 
trova ad affrontare una profonda crisi economica perché non adeguatamente sostenuto dalla politica agricola comune (PAC) dell'Unione europea né da una politica pubblica nazionale ${ }^{6}$.

Significative ed esplicative, al riguardo, sono le "Linee guida per una legge quadro sulle agricolture contadine" (da ora in poi, "Linee guida") presentate nel 2013 al Parlamento italiano dalle organizzazioni promotrici della Campagna, poiché inquadrano le richieste di riconoscimento e di tutela di quello che è considerato un "modello" di agricoltura nell'ambito dell'istanza di "sovranità alimentare dei popoli", intesa come "diritto alla produzione, controllo e gestione del proprio cibo da parte dei contadini e dei cittadini" "7. Punto di partenza del ragionamento politico alla base delle

produttive agricole con meno di 5 ettari di Superficie Agricola Utilizzata (SAU), sono il $71,7 \%$ del totale ma gestiscono solo il $12,6 \%$ della SAU mentre quelle con oltre 20 ettari di SAU rappresentano appena il $9 \%$ del totale a cui però è riconducibile il $64,8 \%$ della SAU". Inoltre, "La piccola dimensione caratterizza principalmente le aziende agricole di persone fisiche: quelle con meno di 1 ettaro sono oltre 270 mila $(58,6 \%)$, mentre quelle tra 1 e 5 ettari sono quasi 170 mila $(36,4 \%)$ ". Occorrerà attendere la pubblicazione dei risultati del Censimento Generale dell'Agricoltura realizzato nel 2020 per conoscere l'evoluzione recente del numero e della tipologia delle aziende italiane. In ogni caso, secondo l'ISTAT, quella italiana è "una realtà complessa e variegata in cui coesistono modelli produttivi e realtà aziendali eterogenei tra loro. Operano nel settore grandi imprese agricole, caratterizzate da un sistema di management e una struttura organizzativa complessa e da un orientamento fortemente integrato con l'industria della trasformazione agricola $\mathrm{e}$ con la grande distribuzione. Allo stesso tempo continuano a essere presenti unità produttive con un'organizzazione di tipo più tradizionale, con relazioni più o meno strutturate e continuative con il mercato e piccoli coltivatori, la cui produzione è principalmente volta all'autoconsumo". V. https://www.istat.it/it/files//2019/12/Struttura-unit\%C3\%A0-economiche-settore-agricolo.pdf [accesso 9 luglio 2021].

${ }^{6}$ Per una critica alla politica agricola comune (PAC) e al modello di sviluppo che essa rappresenta (qualificato in senso negativo come "sviluppi sta"), in quanto favorisce la rendita e l'accentramento fondiario, anziché sostenere il lavoro, v. S. Cabras, Terra e futuro. L'agricoltura contadina ci salverà, Eurilink 2014, spec. cap. 3. Preoccupazioni su questo aspetto sono, del resto, manifestate anche dagli economisti agrari: v., ad esempio, A. Povellato, Prezzo della terra, mobilità fondiaria e riforma della Pac, "Agriregionieuropa" dicembre 2013, https://agriregionieuropa. univpm.it; F. Sotte, Editoriale n. 49 - Il boomerang delle politiche che favoriscono la rendita, “Agriregionieuropa" dicembre 2017. A livello europeo, il Parlamento si è occupato dell' "iniqua distribuzione dei terreni agricoli" a cui corrisponde "un'iniqua distribuzione delle sovvenzioni della PAC" con la Risoluzione del 27 aprile 2017 sulla situazione relativa alla concentrazione dei terreni agricoli nell'UE: come agevolare l'accesso degli agricoltori alla terra (2016/2141(INI)), raccomandando "agli Stati membri una verifica mirata dell'attuazione nazionale della PAC attuale al fine di identificare gli eventuali effetti indesiderati della concentrazione fondiaria" e invitando la Commissione, in fase di elaborazione della PAC post 2020, a elaborare misure volte a contrastare la concentrazione di terreni agricoli e a sostenere le microimprese e le piccole e medie imprese.

${ }^{7}$ Sull'evoluzione della food sovereignity da istanza affermatasi nella società civile a partire dagli anni '90, soprattutto su iniziativa del movimento contadino internazionale La Via Campesina, a concetto accolto dall'ONU, v., per tutti, M. Alabrese, Il regime della food security nel commercio 
richieste è la presa d'atto della "grande diversità italiana di agroecosistemi e di condizioni socio-economiche [che] ha prodotto [...] nel tempo una pluralità di forme economiche, strutture produttive e mercati agricoli"»; questa "ricchezza della diversità delle agricolture" dovrebbe essere posta a "fondamento di politiche agricole differenziate" cambiando quindi l'attuale politica agricola italiana che si presenta "strutturata per sostenere unicamente un modello agroindustriale di agricoltura specializzata e sempre più capitalizzata nell'ambito della competitività del mercato globale", in attuazione della $\mathrm{PAC}^{10}$. In questo contesto, esiste una "presenza capillare" di imprese agricole sul territorio italiano che rappresenta "ancora oggi il presidio più sicuro per la salvaguardia dei territori montani e collinari svolgendo una articolata serie di funzioni tuttora insostituibili" ${ }^{11}$; forme di agricoltura che vengono di solito identificate con termini che enfatizzano singoli aspetti,

agricolo internazionale. Dall 'Havana Charter al processi di riforma dell' Accordo agricolo WTO, Torino 2018, p. 24 ss. (ivi, anche per i richiami alla letteratura di riferimento). Sulle posizioni assunte dall'European Coordination Via Campesina, nel cui contesto si possono inquadrare le richieste della Campagna per la promozione dell'agricoltura contadina, v. https://www.eurovia. org/. Sul tema, v. infra, par. 3.

${ }^{8}$ Tre sono le tipologie di imprese che le Linee guida individuano schematicamente come rappresentative della pluralità di modelli agricoli esistenti in Italia: le "imprese totalmente inserite nel mercato agroindustriale (alta intensità di capitali e tecnologia, filiera commerciale, aree a forte reddito)"; le "aziende di ridotta dimensione economica e fisica che producono con alta intensità di lavoro e bassa capitalizzazione, per mercati di prossimità ma talvolta anche nazionali ed esteri"; le "piccole aziende di autoconsumo e con limitata vendita diretta (bassa intensità tecnologica e scarsi o assenti capitali, territori considerati marginali)" (Linee guida, par. 1).

9 Sono parole tratte dalle Linee guida, par. 3. Si tenga presente che autorevole dottrina giusagraristica già all'inizio del nuovo millennio aveva richiamato l'attenzione sull'"agricoltura plurale", quale espressione di un'agricoltura "più direttamente insediata sul territorio [che] utilizza le risorse del milieu rural per ampliare lo spettro della propria attività, tutelando in senso ampio valori collettivi e condivisi, come quelli dell'ambiente, del paesaggio e delle tradizioni ed offrendo un modello di sviluppo economicamente sostenibile e socialmente coeso" (F. Adornato, Di cosa parliamo quando parliamo di agricoltura, "Agricoltura Istituzioni Mercati" 2004, n. 1, p. 6; idem, Il territorio come destino, "Agricoltura Istituzioni Mercati” 2014, n. 2, p. 4 ss.).

${ }^{10}$ Sull'integrazione del settore agricolo europeo nel sistema agroalimentare, v. A. Jannarelli, Profili del sistema agro-alimentare e agro-industriale. I rapporti contrattuali nella filiera agroalimentare, Bari 2018.

${ }^{11}$ Linee guida, par. 1. Sull'agricoltura contadina come "a land-labour institution", v. J.D. van der Ploeg, The importance of peasant agriculture: a neglected truth, Wageningen 2017, p. 4. Il noto sociologo rurale prosegue sostenendo che "Peasant agriculture can be defined as grounded on a self-controlled resource base. That is to say, the resources needed to produce food, fibre or whatever are largely available in the farm itself. These resources are part of the patrimony of the farming family and pass from one generation to the other. The self-controlled resource base embraces living nature which is embodied in the land, crops, animals and the local eco-system and the capacity of farmers to know, deal with, develop and convert living nature into food" (p. 5). 
come "agricoltura di piccola scala" 12 oppure "agricoltura locale, agricoltura a km 0 "13 $\mathrm{o}$ ancora "agricoltura familiare" 14 , i quali però, secondo i promotori della Campagna, se presi singolarmente, "limitano la comprensione" della realtà socio-economica. Secondo le Linee guida, molteplici e variegati sono infatti gli elementi che caratterizzano le "agricolture contadine" e che quindi una normativa ad hoc dovrebbe valorizzare; essi attengono non solo al lavoro diretto e manuale del conduttore e alla piccola dimensione, ma anche alla territorialità e all'agroecologia ${ }^{15}$.

Ebbene, il disegno di legge n. 2243 recante "norme per la tutela e la valorizzazione dell'agricoltura contadina", che è attualmente in discussione al Senato dopo essere stato approvato dalla Camera dei Deputati, accoglie alcune delle istanze espresse nelle Linee guida ${ }^{16}$ e pone in evidenza il ruolo

${ }^{12} \mathrm{~V}$., in relazione alla prospettiva sia internazionale sia dell'Unione europea, I. Trapè, Small farming e cambiamenti climatici, "Agricoltura Istituzioni Mercati” 2016, n. 2, p. 88 ss.

${ }_{13}$ V., in proposito, I. Canfora, Dalla terra al territorio: il ruolo dell'agricoltore nella filiera corta, in F. Giarè, S. Giuca (a cura di), Agricoltori e filiera corta. Profili giuridici e dinamiche socioeconomiche, Roma 2012, pp. 37-38, laddove osserva in modo condivisibile che "gli strumenti di promozione della filiera corta in agricoltura permettono di raggiungere quelle finalità della politica agricola indicate all'art. 33 del Trattato (ora 39 TFUE), tra loro difficilmente compatibili, e la cui convergenza è persino considerata favorevolmente ai fini dell'esenzione dell'assoggettamento alle regole della concorrenza per gli accordi adottati nel settore agricolo: la vendita diretta dei prodotti agricoli nella filiera corta, infatti, garantisce l'incremento della produttività, il miglioramento del reddito degli agricoltori, la stabilizzazione dei mercati, la sicurezza degli approvvigionamenti e infine assicura prezzi ragionevoli ai consumatori”.

${ }^{14}$ Per un esame dell'agricoltura familiare come fonte principale di produzione di cibo nel mondo e, quindi, della famiglia agricola come "soggetto" da proteggere e promuovere nell'ottica della global food security e della protezione della natura, pur constatandone l'assenza di una nozione universalmente condivisa, v. FAO, The State of Food and Agriculture. Innovation in Family Farming, Roma 2014 (http://www.fao.org/3/a-i4040e.pdf). Dati relativi alla consistenza e all'evoluzione dell'agricoltura familiare nell'Unione europea sono reperibili dal documento commissionato dal Parlamento europeo dal titolo Family Farming in Europe: Challenges and Prospects. In-Depth Analysis, 2014, https:/www.europarl.europa.eu/RegData/etudes/note/ join/2014/529047/IPOL-AGRI_NT(2014)529047_EN.pdf [accesso 22 luglio 2021]. Sui dati relativi alla situazione italiana, emergente dal Censimento generale dell'agricoltura del 2010, v. INEA, Agricoltura familiare tra lavoro e stile di vita, tra produzione e sviluppo, Roma 2014. Per un analisi interdisciplinare del fenomeno, cfr. il fascicolo n. 43 di "Agriregionieuropa" 2015, interamente dedicato al tema dell'agricoltura familiare.

${ }^{15}$ Sui profili di interesse giuridico del paradigma dell'agroecologia, v., per tutti, M. Monteduro, "Ius et rus": la rilevanza dell'agroecologia per il diritto, "Rivista quadrimestrale di diritto dell'ambiente" 2019 , n. 2, p. 4 ss., anche per l'ampia letteratura a carattere interdisciplinare ivi citata.

${ }^{16}$ Le prime proposte di legge sull'agricoltura contadina sono state presentate e discusse alla Commissione agricoltura della Camera dei deputati durante la XVII Legislatura (v., in proposito, F. Adornato, Le agricolture contadine, “Agricoltura Istituzioni Mercati” 2016, n. 1, p. 5 ss.; I. Trapè, Small farming..., p. 104 ss.; I. Giunta, La Campagna Popolare per l'Agricoltura Contadina e le proposte per una legge di tutela, "Agriregionieuropa", giugno 2016; A. Onorati, 
strumentale dell' agricoltura contadina rispetto agli obiettivi della promozione dell'agroecologia ${ }^{17}$ (inteso come metodo di produzione attento alla salvaguardia dei terreni, alla biodiversità animale e vegetale, alla qualità delle produzioni agricole, al rispetto e alla protezione del suolo, nonché ai valori delle culture tradizionali e del territorio ${ }^{18}$ ) e della lotta allo spopolamento delle zone marginali sia di pianura e periurbane che montane e collinari ${ }^{19}$.

Il riconoscimento dell'agricoltura contadina e degli agricoltori che la praticano passa attraverso l'istituzione di un 'Registro dell'agricoltura contadina", a valenza nazionale, nel quale possono iscriversi gratuitamente le "aziende agricole contadine" e "gli agricoltori contadini" è esplicitato, ma senz'altro, oltre all'importante funzione di censire e dare visibilità e dignità autonoma a questo tipo di agricoltura, che è espressione anche di un modo di vivere ${ }^{21}$, consiste nel permettere di ottenere $i$ benefici previsti da norme di favore previste dal medesimo disegno di legge, fra cui la preferenza nell'assegnazione di terreni incolti o abbandonati iscritti nelle "Banche della terra" regionali ${ }^{22}$ oppure l'attribuzione di un punteggio premiale nell'ambito di misure di sviluppo rurale eventualmente previste dal Piano strategico nazionale italiano in applicazione della PAC post-202123.

In effetti, appare singolare la scelta di prevedere un Registro riservato sia a soggetti che svolgono l'attività agricola connotata come "contadina" (gli "agricoltori contadini”) sia alle aziende, ovvero a complessi di beni or-

Agricoltura italiana..., spec. p. 95 ss.). Nel corso dell'attuale XVIII Legislatura le proposte sono state ripresentate e riunificate in un unico disegno di legge, che è stato approvato dalla Camera dei deputati il 20 maggio 2021 e poi trasmesso al Senato. Attualmente (7 luglio 2021) è in corso di esame da parte della Commissione "Agricoltura e produzione agroalimentare": v. il sito del Senato della Repubblica http://www.senato.it/leg/18/BGT/Schede/Ddliter/54101. htm [accesso 9 luglio 2021].

${ }^{17}$ L'art. 1, comma 3, del ddl n. 2243 richiama il documento della FAO relativo ai "Dieci elementi dell'agroecologia", approvato dal Consiglio della FAO il 3 dicembre 2019 (v. FAO, The 10 Elements of Agroecology. Guiding the transition to sustainable food and agricultural systems: http://www.fao.org/agroecology/overview/overview10elements/en/). Tali “elementi" sono: Diversity, Co-creation and sharing of knowledge, Synergies, Efficiency, Recycling, Resilience, Human and Social Value, Culture and Food Tradition, Responsible governance, Circular and solidarity economy.

18 V. l'art. 1 "Oggetto e finalità", spec. comma 3, lettere a) e c) del ddl n. 2243.

19 V. l'art. 1, citato, spec. comma 3, lettere d) e e) del ddl n. 2243.

20 V. l'art. 3 del ddl n. 2243.

${ }^{21}$ J.D. van der Ploeg, The New Peasantries: Struggles for Autonomy and Sustainability in an Era of Empire and Globalization, London 2008, p. 40, parla di "condizione contadina", come ricorda I. Trapè, Small farming..., p. 103

${ }^{22}$ V. l'art. 6 del ddl n. 2243.

${ }_{23}$ V. l'art. 5 del ddl n. 2243. 
ganizzati dagli imprenditori agricoli per l'esercizio dell'impresa ${ }^{24}$, sempre secondo il modello dell'agricoltura contadina (appunto, le "aziende agricole contadine"). Prescindendo da ogni considerazione sulla scelta terminologica, che predilige il riferimento al profilo oggettivo dell'azienda anziché a quello soggettivo dell'imprenditore, l'intenzione sembra quella di voler distinguere all'interno della categoria dei contadini coloro che sono giuridicamente qualificabili come imprenditori (e che quindi detengono un'organizzazione aziendale) da coloro che, pur esercitando l'attività agricola, imprenditori non sono. Ciò è espressione del rifiuto, manifestato dai promotori della Campagna per l'agricoltura contadina, nei confronti del paradigma unitario e unificatore dell'impresa agricola di impostazione codicistica che ha finito per penalizzare e "oscurare" la realtà diversificata e complessa dell'agricoltura italiana di piccola dimensione, niente affatto scomparsa ${ }^{25}$. Essa comprende, infatti, anche soggetti che lavorano la terra ${ }^{26}$ per autoconsumo e che ricorrono alla vendita diretta per ottenere un reddito che garantisca la sopravvivenza, non quindi un capitale da reinvestire ${ }^{27}$.

Il disegno di legge definisce le "aziende agricole contadine" come le aziende agricole che rispondono ai seguenti requisiti: sono condotte direttamente dal titolare, dai familiari o dai soci di una cooperativa di lavoratori, attraverso il loro apporto di lavoro prevalente sia con riguardo al tempo dedicato alla produzione contadina sia con riguardo all'eventuale collaborazione di lavoratori stagionali o di dipendenti fissi; in esse si praticano "modelli di produzione agroecologica", in conformità con le "Linee guida volontarie sulla gestione responsabile della terra, dei territori di pesca

${ }^{24}$ Così, secondo il combinato disposto di cui agli articoli 2135 (sull'imprenditore agricolo) e 2555 (sull'azienda agricola) del codice civile italiano.

${ }^{25}$ Tra virgolette si riporta il termine utilizzato da F. Adornato, Le agricolture contadine..., p. 6, laddove con riferimento al codice civile del 1942 parla di "logica unificatrice e avvolgente dell'impresa agricola [che] aveva oscurato i fenomeni plurali dell'agricoltura", evidenziando come la dimensione dell'agricoltura plurale è oggi alla base del fenomeno delle agricolture contadine (di cui ai progetti di legge della XVII Legislatura italiana).

${ }^{26}$ Cfr., in proposito, A. Onorati, Agricoltura italiana..., p. 87. Coerentemente con il pensiero esposto nel testo, merita evidenziare come, sotto il profilo terminologico, i promotori della Campagna preferiscono parlare di "contadini" anziché di imprenditori agricoli e di "lavoro della terra" o nei campi, anziché di coltivazione o genericamente di attività agricola, per sottolineare l'impegno diretto (e faticoso) degli agricoltori che non è diretto al "mercato", identificato con la grande distribuzione organizzata.

${ }^{27} \mathrm{Si}$ tratta spesso di aziende che rientrano in quelle situazioni in cui l'ordinamento italiano prevede l'esonero del pagamento dell'imposta sul valore aggiunto. Cfr., in proposito, il Dossier del Servizio Studi della Camera dei Deputati, Fiscalità agricola, misure per i giovani e previdenza, 8 giugno 2021, https://www.camera.it/temiap/documentazione/temi/pdf/1208657. pdf?_1591370973480 [accesso 23 luglio 2021]. 
e delle foreste" della FAO ${ }^{28}$ e con il documento della FAO sui "Dieci elementi dell'agroecologia" 29 ; favoriscono la tutela e la conservazione del territorio nei suoi aspetti ambientali e paesaggistici fondamentali; trasformano le materie prime prodotte nelle aziende senza effettuare lavorazioni in serie prevalentemente automatizzate, privilegiando forme di economia solidale e partecipata ${ }^{30}$; producono limitate quantità di beni agricoli e alimentari, destinati al consumo immediato e alla vendita diretta ai consumatori finali ${ }^{31}$; rientrano nella disciplina del coltivatore diretto, di cui all'art. 2083 del codice civile italiano $^{32}$, oppure delle forme associative o cooperative ${ }^{33}$.

Sono, invece, definiti "agricoltori contadini" "i proprietari o i conduttori di terreni agricoli che esercitano su di essi attività agricole non in via principale", secondo i modelli di produzione agroecologici e trasformando le materie prime prodotte in azienda secondo quanto prescritto nell'ambito della definizione di "aziende agricole contadine"34.

Ciò significa che, nell'ambito del genus agricoltori, individuabili in base alla teoria agro-biologica come coloro che curano il ciclo biologico di un essere vegetale o animale ${ }^{35}$, è qualificata la species contadini, in virtù di requisiti di carattere oggettivo e soggettivo che valorizzano il rapporto con

${ }^{28}$ The Voluntary Guidelines on the Responsible Governance of Tenure of Land, Fisheries and Forests in the Context of National Food Security sono consultabili sul sito della FAO: http://www. fao.org/tenure/voluntary-guidelines/en/. All'esame del punto di vista degli Stati europei rispetto a tali Linee guida è stata dedicata la Commissione I dell'ultimo Congresso dell'European Council For Rural Law - CEDR (XXX Congresso, 18-21 settembre 2019, Poznań), nell'ambito del tema "Food Sovereignity and Food Security: Concepts and Legal Framework", i cui papers sono reperibili sul sito del CEDR: https://www.cedr.org/events/2019-congress-poznan/ [accesso 22 luglio 2021].

${ }^{29}$ V. supra, nota 17.

${ }^{30}$ Sull'economia partecipata e sulla certificazione partecipata, v. S. Cabras, Terra e futuro..., cap. 3, par. 3, e, con riferimento all'esperienza di Slow food, S. Rolandi, Slow Food e il progetto dei Presidi. Verso l'adozione di sistemi partecipativi di garanzia?, in A. Di Lauro, G. Strambi (a cura di), Le funzioni sociali dell'agricoltura, Pisa 2020, p. 212 ss. (ivi, anche per specifiche indicazioni bibliografiche).

${ }^{31}$ La disciplina italiana della vendita diretta è dettata dall'art. 4 del d. lgs. 18 maggio 2001, n. 228. Sul tema, v., di recente, A. Kapała, Legal Instruments to Support Local Food Systems in Italian Law, "EU Agrarian Law" 2020, vol. 9, p. 5 ss.; M. Alabrese, Sobre la legislación italiana para el fomento de cadena corta en la venta de productos alimenticios, "Revista Iberoamericana de Derecho Agrario" 2017, n. 6.

${ }^{32}$ L'art. 2083 del codice civile italiano recita così:"Sono piccoli imprenditori i coltivatori diretti del fondo, gli artigiani, i piccoli commercianti e coloro che esercitano un'attività professionale organizzata prevalentemente con il lavoro proprio e dei componenti della propria famiglia".

33 V. 1'art. 2, comma 1 del ddl n. 2243.

${ }^{34}$ V. l'art. 2, comma 2 del ddl n. 2243.

${ }^{35}$ Come è noto, la teoria che rinviene il fondamento dell'agrarietà nel ciclo biologico di un essere vegetale o animale è stata formalizzata da A. Carrozza, Problemi generali e profili di qualificazione del diritto agrario, Milano 1975. 
la risorsa naturale-terra e con il territorio, indipendentemente dall'esercizio dell'attività in forma di impresa ex art. 2082 del codice civile italiano.

Il disegno di legge prevede, inoltre, che spetti alle Regioni dettare la disciplina specifica sulla produzione, trasformazione e vendita dei prodotti dell'agricoltura contadina, che semplifichi le norme igieniche e sanitarie pur garantendo un livello elevato di tutela della salute dei consumatori, nonché le procedure agevolate per l'esecuzione di lavori, anche in economia, di manutenzione ordinaria e straordinaria degli edifici rurali dell'azienda sia per uso abitativo sia come annessi agricoli, seppure nel rispetto dei principi che saranno stabiliti con un apposito decreto del Ministro delle politiche agricole alimentari e forestali ${ }^{36}$.

Infine, merita richiamare, per il suo valore simbolico, l'istituzione della Giornata nazionale dedicata alla cultura e alle tradizioni dell'agricoltura contadina nel giorno dell'11 novembre ${ }^{37}$, corrispondente all'inizio dell'annata agraria ai sensi dell'art. 39 della legge 3 maggio 1982, n. 203 recante "Norme sui contratti agrari".

\section{Le istanze globali: la Dichiarazione ONU sui diritti dei contadini e delle altre persone che lavorano nelle zone rurali}

Il disegno di legge sulla tutela e valorizzazione dell'agricoltura contadina richiama in apertura, tra i principi ispiratori, la Dichiarazione sui diritti dei contadini e delle altre persone che lavorano in ambito rurale, adottata

${ }^{36}$ Così, l'art. 4 del ddl n. 2243. Merita ricordare, al riguardo, che alcune Regioni italiane hanno già legiferato per sostenere indirettamente l'agricoltura contadina (intesa come attività di produzione a livello locale e di piccola scala) avvalendosi dei confini flessibili concessi dalle norme dell'Unione europea in materia di igiene degli alimenti per semplificare i requisiti strutturali e igienico-sanitari dei locali e delle attrezzature. V., ad esempio, la legge della Regione Toscana 21 marzo 2018, n. 12, "Disposizioni per la lavorazione, la trasformazione ed il confezionamento dei prodotti agricoli di esclusivamente provenienza aziendale" e il successivo regolamento di attuazione, Decreto del Presidente della Giunta regionale 24 luglio 2019, n. 46/R (reperibile sul sito http://raccoltanormativa. consiglio.regione.toscana.it/documenti-tree/leggi/), su cui v. S. Carmignani, L'agricoltura contadina nella legge Regione Toscana 21 marzo, n. 12, "Studi senesi" 2019, p. 403 ss.

${ }^{37}$ V. 1'art. 9 del ddl n. 2243. Per completezza, occorre segnalare che il disegno di legge comprende una serie di articoli dedicati al recupero dei terreni incolti e abbandonati e alla tutela del suolo a scopi agricoli, ma non è qui possibile entrare nel dettaglio di queste norme, che attengono profili di interesse che vanno oltre il tema dell'agricoltura contadina, pur affrontando un'importante questione ambientale, paesaggistica e sociale, ancora irrisolta per l'Italia. Sull'attualità di tale problematica, v. G. Strambi, La questione delle terre incolte e abbandonate e le leggi sulle "banche della terra", "Rivista di diritto agrario" 2017, n. 1, p. 599 ss. 
dall'Assemblea Generale dell'ONU il 17 dicembre 2018 (da ora in poi, Dichiarazione $)^{38}$, a cui le finalità del provvedimento sono dichiarate conformi. Tale richiamo è particolarmente significativo per due ordini di motivi: da un lato, perché riflette un cambiamento della posizione politica dello Stato italiano, il quale si astenne in occasione del voto finale di approvazione della Dichiarazione, dimostrando, invece, adesso una preoccupazione per le condizioni di vita dei contadini anche nel nostro Paese ${ }^{39}$; dall'altro, perché implica la volontà di conformarsi alle raccomandazioni espresse in uno strumento di tutela dei diritti umani che riconosce un ruolo centrale all'agricoltura di piccola scala, a carattere essenzialmente familiare e fondata su conoscenze tradizionali, nel perseguimento non solo del miglioramento delle condizioni di vita nelle aree rurali, ma anche degli obiettivi della sicurezza alimentare e della lotta ai cambiamenti climatici, e che, a tal fine, incoraggia gli Stati a sostenere un'agricoltura sostenibile, che comprende quella biologica e quella agro-ecologica ${ }^{40}$.

Pur senza entrare nel dettaglio del contenuto della Dichiarazione ${ }^{41}$, vale la pena ricordarne alcuni aspetti essenziali, rilevanti per la migliore comprensione del contesto in cui è maturato il disegno di legge domestico. Innanzitutto,

${ }^{38}$ L'art. 1 del ddl n. 2243 richiama, innanzitutto, l'art. 44 della Costituzione italiana e, in seguito, altri atti internazionali: la Convenzione sulla biodiversità del 1992; il Trattato internazionale sulle risorse fitogenetiche per l'alimentazione e l'agricoltura del 2001; le Linee guida volontarie della FAO sulla gestione responsabile della terra, dei territori di pesca e delle foreste del 2012; i già citati Dieci elementi dell'agroecologia, FAO, 2019.

39 Sul processo di negoziazione della Dichiarazione ONU e sull'evoluzione della posizione della maggior parte degli Stati membri dell'UE (inclusa l'Italia) da una iniziale opposizione al progetto alla decisione di astensione nella votazione finale, v. C. Hubert, The United Nations Declaration on the Rights of Peasants: A Tool in the Struggle for our Common Future, Geneva 2019, Part I. Si tenga presente, peraltro, che il Parlamento europeo, con la European Parliament Resolution of 3 July 2018 on violation of the rights of indigenous peoples in the world, including land grabbing (2017/2206(INI)), aveva invitato l'Unione europea e gli Stati membri a supportare e votare in favore della Dichiarazione. Invito che, evidentemente, è stato disatteso.

${ }^{40}$ V. l'art. 16, par. 4, della Dichiarazione ONU. Cfr., in proposito, L. Paoloni, S. Vezzani, $L a$ Dichiarazione ONU sui diritti dei contadini e delle altre persone che lavorano nelle aree rurali: prime riflessioni, "federalismi.it" $1^{\circ}$ maggio 2019, p. 27; E. Cristiani, G. Strambi, Farming Models and Peasants' Rights, in: M. Alabrese, A. Bessa, M. Brunori, P.F. Giuggioli (a cura di), Peasants' Rights The United Nations Declaration on the Rights of Peasants and other people working in rural areas in perspective (in corso di pubblicazione).

${ }^{41}$ Su cui v. L. Paoloni, S. Vezzani, La Dichiarazione ONU..., p. 2 ss.; i contributi di M. Alabrese, A. Di Lauro, O. Ferrajolo, in: A. Di Lauro, G. Strambi (a cura di), Le funzioni sociali dell'agricoltura, Pisa 2020; M.A. Victoria, Nuevos derechos humanos de los campesinos en el marco de la Declaración de Naciones Unidas de 2018, "Revista Iberoamericana de Derecho Agrario" marzo 2020; e, da ultimo, M. Alabrese, A. Bessa, M. Brunori, P.F. Giuggioli (a cura di), Peasants'Rights The United Nations Declaration... 
essa parla di peasants e non di farmers ${ }^{42}$, definendoli come "any person who engages or who seeks to engage, alone, or in association with others or as a community, in small-scale agricultural production for subsistence and/or for the market, and who relies significantly, though not necessarily exclusively, on family or household labour and other non-monetized ways of organizing labour, and who has a special dependency on and attachment to the land"43. Tra i diritti dei contadini (e delle altre persone che lavorano in zone rurali ${ }^{44}$ ) la Dichiarazione richiama quello alla sovranità alimentare, laddove prevede il diritto dei contadini di determinare i propri sistemi alimentari e agricoli, inclusi il diritto di partecipare ai processi decisionali sulle politiche alimentari e agricole e il diritto ad alimenti sani e adeguati, prodotti tramite metodi ecologicamente sicuri e sostenibili che rispettano le loro culture ${ }^{45}$. Un diritto che mette in discussione le logiche appropriative e distributive delle risorse naturali proprie del paradigma agroalimentare globale oggi dominante e che fa da collante all'affermazione di "diritti vecchi e nuovi"

${ }^{42}$ Osservazione di L. Paoloni, S. Vezzani, La Dichiarazione ONU..., p. 11.

${ }^{43}$ Art. 1, par. 1, della Dichiarazione. Sulla nozione di peasants nella Dichiarazione, v. L. Paoloni, S. Vezzani, La Dichiarazione ONU..., par. 3.

${ }^{44}$ L'art. 1, par. 2,3 e 4, della Dichiarazione ONU recita così: "2. The present Declaration applies to any person engaged in artisanal or small-scale agriculture, crop planting, livestock raising, pastoralism, fishing, forestry, hunting or gathering, and handicrafts related to agriculture or a related occupation in a rural area. It also applies to dependent family members of peasants. 3 . The present Declaration also applies to indigenous peoples and local communities working on the land, transhumant, nomadic and semi-nomadic communities, and the landless engaged in the above-mentioned activities. 4. The present Declaration further applies to hired workers, including all migrant workers regardless of their migration status, and seasonal workers, on plantations, agricultural farms, forests and farms in aquaculture and in agro-industrial enterprises".

${ }^{45}$ Art. 15 della Dichiarazione ONU, su cui v. L. Paoloni, S. Vezzani, La Dichiarazione ONU..., p. 28; A. Di Lauro, La sostenibilità nella Dichiarazione dei diritti dei contadini. Diritti bioculturali e sovranità alimentare, in: A. Di Lauro, G. Strambi (a cura di), Le funzioni sociali dell'agricoltura, Pisa 2020, p. 67 ss.; M. Alabrese, Le sfide sociali dell'agricoltura: la transizione verso sistemi agroalimentari sostenibili, ibidem, par. 3.

${ }^{46}$ L. Paoloni, S. Vezzani, La Dichiarazione ONU..., p. 12. Senza pretesa di esaustività, i diritti e le libertà dei contadini enunciati dalla Dichiarazione sono: il diritto all'accesso e all'uso sostenibile delle risorse naturali presenti nelle loro comunità; il diritto alla vita, all'integrità fisica e mentale, alla libertà e alla sicurezza della persona; la libertà di movimento; la libertà di pensiero, religione, espressione; la libertà di riunirsi in sindacati, cooperative o altre associazioni; il diritto di partecipazione libera e attiva alla preparazione e implementazione di politiche e programmi che hanno un impatto sulla loro vita; il diritto all'informazione anche per ciò che concerne la produzione e commercializzazione dei loro prodotti; il diritto all'accesso alla giustizia e all'assistenza giuridica; il diritto al lavoro e alla sicurezza sul lavoro; il diritto al cibo e alla sovranità alimentare; il diritto a un tenore di vita adeguato e all'accesso facilitato ai mezzi di sostentamento necessari per ottenerlo; il diritto alla terra; il diritto alla conservazione e alla protezione dell'ambiente e delle capacità produttive delle loro terre e risorse; il diritto alle sementi; il diritto all'acqua pulita; il diritto alla 
zione, come il diritto al lavoro e alla sicurezza sul lavoro, il diritto ad una alimentazione adeguata e ad essere liberi dalla fame, da un lato, e il diritto alla terra e il diritto alle sementi, dall'altro ${ }^{47}$.

Strategico per garantire il diritto ad un tenore di vita adeguato ai contadini e alle loro famiglie è, peraltro, non solo l'accesso ai mezzi di produzione, ma anche l'accesso ai mercati locali, nazionali e regionali. A tal fine è significativo che la Dichiarazione raccomandi agli Stati di adottare misure che proteggano e rafforzino le opportunità di sostentamento locale che includono la "facilitazione" della vendita diretta dal produttore al consumatore ${ }^{48}$; quella vendita diretta su cui non a caso focalizza l'attenzione anche il legislatore italiano.

\section{Conclusioni}

In questa epoca di pandemia COVID-19 è emerso a vari livelli (nazionale, regionale e internazionale) il ruolo strategico del settore agro-alimentare ${ }^{49}$. Al contempo, il sistema alimentare fondato sulle grandi multinazionali e in generale sul modello dell'agribusiness ha mostrato chiaramente profonde criticità, rispetto alle quali i sistemi agricoli locali basati sulle filiere corte hanno svolto un ruolo di supplenza ${ }^{50}$.

previdenza sociale; il diritto alla salute fisica e mentale; il diritto all'alloggio adeguato; il diritto all'istruzione; il diritto di godere della propria cultura e di mantenere, proteggere e sviluppare le proprie conoscenze tradizionali.

${ }^{47}$ Sui profili di conflittualità che ancora rimangono aperti intorno al diritto alla terra e il diritto alle sementi come enunciati nella Dichiarazione, v. C. Hubert, The United Nations Declaration on the Rights of Peasants..., part II; C. Golay, A. Bessa, The United Nations Declaration on the Rights of Peasants and Other People Working in Rural Areas and the Protection of the Right to Seeds in Europe, Geneva Academy of International Humanitarian Law and Human Rights, Academy Briefing No. 15, Ginevra, April 2019.

${ }^{48}$ V. 1'art. 16, par. 4, della Dichiarazione.

${ }^{49}$ V. la Comunicazione della Commissione UE, "Una strategia 'Dal produttore al consumatore' per un sistema alimentare equo, sano e rispettoso dell'ambiente”, COM (2020) 381, del 20 maggio 2020, p. 2, laddove riconosce che "La pandemia di COVID-19 ha sottolineato l'importanza di un sistema alimentare solido e resiliente che funzioni in qualsiasi circostanza e sia in grado di assicurare ai cittadini un approvvigionamento sufficiente di alimenti a prezzi accessibili”.

${ }_{50}$ M. Alabrese, Le sfide sociali dell'agricoltura..., p. 79, ritiene, invero, insufficienti i pochi cenni alle filiere corte e ai sistemi alimentari regionali e locali che la Commissione europea fa nel documento su "La strategia 'Dal produttore al consumatore'...", citata. Secondo l'A., infatti, "la visione generale rimane ancora fortemente orientata verso la filiera alimentare globalizzata", mostrando "scarsa considerazione per i piccoli agricoltori che pure, a seguito della pandemia COVID-19, si sono dimostrati essenziali anche in Europa e la cui funzione sociale è indubbia ..." (ibidem, p. 80). Sul mutamento delle modalità di consumo dei cittadini italiani durante il periodo di 
Il contesto appare quindi propizio per favorire un cambiamento di paradigma anche nei Paesi cosiddetti sviluppati, tra i quali gli Stati membri dell'Unione europea, orientato verso un impegno di questi ultimi e delle Istituzioni dell'Unione nel garantire non solo la sopravvivenza, ma anche la valorizzazione del ruolo dei piccoli agricoltori per la food security, implementando la Dichiarazione ONU sui diritti dei contadini ${ }^{51}$. Essi, del resto, secondo le stime dalla $\mathrm{FAO}$, già contribuiscono, in media, a produrre il $70 \%$ del cibo nel mondo; una percentuale che sale oltre 1' $80 \%$ nei Paesi in via di sviluppo ${ }^{52}$.

Alla luce di ciò, il disegno di legge sull'agricoltura contadina può essere apprezzato come un primo passo verso il riconoscimento di soggetti del settore primario che ambiscono ad acquisire maggiore importanza nella programmazione di politiche agricole e di coesione nazionali (e dell'Unione europea) nell'ottica, appunto, della sicurezza alimentare, nonché della lotta al cambiamento climatico, in virtù delle pratiche sostenibili da essi adottate. Uno step che, però, indipendentemente dall'approvazione definitiva della legge, non può che indurre i giusagraristi italiani ad aprire una riflessione sull'attuale sistema di figure giuridiche soggettive in agricoltura, in considerazione delle sollecitazioni che provengono dalle analisi economiche e sociologiche, solo parzialmente richiamate nel presente contributo.

In particolare, sorgono spunti di riflessione intorno alla figura del piccolo imprenditore in agricoltura, appiattita su quella di cui all'art. 2083 del codice civile, nonché sulla generale opportunità di introdurre nell'ordinamento italiano nuove definizioni (come quella di "contadino"), che prescindono dalla nozione di imprenditore ex art. 2082 codice civile ${ }^{53}$.

lockdown, v. M. Gioia, Abitudini alimentari e filiere resilienti nel lockdown da Covid-19, "Rivista di diritto alimentare" 2021, n. 1, p. 78 ss.

${ }^{51}$ Cfr., in proposito, Joint statement by UN human rights experts - $1^{\text {st }}$ anniversary of the adoption of the UN Declaration on the Rights of Peasants and Other People Working in Rural Areas. The need to take steps to implement the UN Declaration on the rights of peasants and other people working in rural areas, 19 Dicembre 2019 (consultabile su https://www.ohchr.org/ EN/NewsEvents/Pages/DisplayNews.aspx?NewsID=25439\&LangID=E; accesso 23 luglio 2021).

${ }_{52}$ Per l'esame della piccola agricoltura alla luce dell'evoluzione del dibattito sulla sicurezza alimentare, v. di recente G. Brunori et al., Il ruolo delle piccole aziende agricole europee nei sistemi alimentari sostenibili e nella sicurezza alimentare e nutrizionale, in: A. Di Lauro, G. Strambi (a cura di), Le funzioni sociali dell'agricoltura, Pisa 2020, p. 163 ss.

${ }_{53}$ Giudica, ad esempio, non opportuna l'introduzione di una nuova figura giuridica soggettiva nell'ordinamento italiano, I. Trapè, Small farming..., p. 105, ritenendo “preferibile un 'aggiornamento' delle figure soggettive", come ad esempio del piccolo imprenditore di cui all'art. 2083 del codice civile, che "oggi è tarato esclusivamente sul coltivatore diretto". Ritiene, invece, che "il mutato quadro economico e sociale, non solo a livello internazionale ma anche nazionale [...] può indurre ad un ampliamento delle maglie della figura del coltivatore diretto, la più vicina, appunto, a quella di 
Ulteriori sollecitazioni potranno provenire, inoltre, dall'esito del processo di elaborazione della PAC post 2021 e dalla capacità degli Stati membri, in fase di adozione dei Piani strategici nazionali, di cogliere e soddisfare le istanze locali, che sono espressione delle plurime agricolture territoriali.

\section{BIBLIOGRAFIA}

Adornato F. (2004), Di cosa parliamo quando parliamo di agricoltura, "Agricoltura Istituzioni Mercati" n. 1.

Adornato F. (2014), Il territorio come destino, “Agricoltura Istituzioni Mercati” n. 2.

Adornato F. (2016), Le agricolture contadine, "Agricoltura Istituzioni Mercati” n. 1.

Alabrese M. (2017), Sobre la legislación italiana para el fomento de cadena corta en la venta de productos alimenticios, "Revista Iberoamericana de Derecho Agrario" n. 6.

Alabrese M. (2018), Il regime della food security nel commercio agricolo internazionale. Dall'Havana Charter al processi di riforma dell'Accordo agricolo WTO, Torino.

Alabrese M. (2020), Le sfide sociali dell'agricoltura: la transizione verso sistemi agroalimentari sostenibili, in A. Di Lauro, G. Strambi (a cura di), Le funzioni sociali dell'agricoltura, Pisa.

Brunori G. et al. (2020), Il ruolo delle piccole aziende agricole europee nei sistemi alimentari sostenibili e nella sicurezza alimentare e nutrizionale, in A. Di Lauro, G. Strambi (a cura di), Le funzioni sociali dell'agricoltura, Pisa.

Budzinowski R. (2013), Il diritto agrario di fronte alle sfide contemporanee. Alcune riflessioni, "Agricoltura Istituzioni Mercati" n. 1-2.

Budzinowski R. (2016), Considerazioni sullo sviluppo del diritto agrario (Riflessioni intorno ai diritti della terra e del mercato agroalimentare), in: I diritti della terra e del mercato agroalimentare - Liber amicorum Alberto Germanò, t. II, Milano.

Budzinowski R. (2018), Contemporary challenges of agricultural law: among globalization, regionalization and locality (introductory considerations), "Przegląd Prawa Rolnego" n. 1.

Cabras S. (2014), Terra e futuro. L'agricoltura contadina ci salverà, Eurilink.

Canfora I. (2012), Dalla terra al territorio: il ruolo dell'agricoltore nella filiera corta, in F. Giarè, S. Giuca (a cura di), Agricoltori e filiera corta. Profili giuridici e dinamiche socio-economiche, Roma.

Carmignani S. (2019), L'agricoltura contadina nella legge Regione Toscana 21 marzo, n. 12, "Studi senesi".

Carrozza A. (1975), Problemi generali e profili di qualificazione del diritto agrario, Milano. Cristiani E. (2019), Quali regole per un'agricoltura sostenibile, "Rivista di diritto agrario" n. 1.

contadino", L. Paoloni, I diritti dei contadini e la Costituzione, in: E. Cristiani, A. Di Lauro, E. Sirsi (a cura di), Agricoltura e Costituzione. Per una Costituzione dell'agricoltura, Pisa 2019, p. 260, la quale sostiene che la Costituzione italiana, pur non menzionando manifestamente il termine "contadini", all'art. 44 "rappresenta comunque le esigenze delle comunità rurali che necessitano di stabilire equi rapporti sociali e di conseguire un razionale sfruttamento del suolo oltre che di accedere all'uso del bene terra" (p. 259). 
Cristiani E., Strambi G., Farming Models and Peasants'Rights, in: M. Alabrese, A. Bessa, M. Brunori, P.F. Giuggioli (a cura di), Peasants'Rights The United Nations Declaration on the Rights of Peasants and other people working in rural areas in perspective (in corso di pubblicazione).

Di Lauro A. (2020), La sostenibilità nella Dichiarazione dei diritti dei contadini. Diritti bioculturali e sovranità alimentare, in: A. Di Lauro, G. Strambi (a cura di), Le funzioni sociali dell'agricoltura, Pisa.

Di Lauro A., Strambi G. (a cura di) (2020), Le funzioni sociali dell'agricoltura, Pisa.

Gioia M. (2021), Abitudini alimentari e filiere resilienti nel lockdown da Covid-19, "Rivista di diritto alimentare" n. 1.

Giunta I. (2016), La Campagna Popolare per l'Agricoltura Contadina e le proposte per una legge di tutela, "Agriregionieuropa" giugno.

Golay C., Bessa A. (2019), The United Nations Declaration on the Rights of Peasants and Other People Working in Rural Areas and the Protection of the Right to Seeds in Europe, Geneva Academy of International Humanitarian Law and Human Rights, Academy Briefing No. 15, Ginevra, April.

Hubert C. (2019), The United Nations Declaration on the Rights of Peasants: A Tool in the Struggle for our Common Future, part. I, Geneva.

Jannarelli A. (2018), Profili del sistema agro-alimentare e agro-industriale. I rapporti contrattuali nella filiera agro-alimentare, Bari.

Kapała A. (2020), Legal Instruments to Support Local Food Systems in Italian Law, "EU Agrarian Law" vol. 9.

Monteduro M. (2019), "Ius et rus": la rilevanza dell'agroecologia per il diritto, "Rivista quadrimestrale di diritto dell'ambiente" n. 2.

Onorati A. (2017), Agricoltura italiana e agricoltura contadina. La necessità di un quadro giuridico specifico, "Parole chiave" n. 2.

Paoloni L. (2019), I diritti dei contadini e la Costituzione, in: E. Cristiani, A. Di Lauro, E. Sirsi (a cura di), Agricoltura e Costituzione. Per una Costituzione dell'agricoltura, Pisa.

Paoloni L., Vezzani S. (2019), La Dichiarazione ONU sui diritti dei contadini e delle altre persone che lavorano nelle aree rurali: prime riflessioni, "federalismi.it" $1^{\circ}$ maggio.

Ploeg J.D. van der (2008), The New Peasantries: Struggles for Autonomy and Sustainability in an Era of Empire and Globalization, London.

Ploeg J.D. van der (2017), The importance of peasant agriculture: a neglected truth, Wageningen.

Povellato A. (2013), Prezzo della terra, mobilità fondiaria e riforma della Pac, "Agriregionieuropa" dicembre.

Rolandi S. (2020), Slow Food e il progetto dei Presidi. Verso l'adozione di sistemi partecipativi di garanzia?, in: A. Di Lauro, G. Strambi (a cura di), Le funzioni sociali dell'agricoltura, Pisa.

Sotte F. (2017), Editoriale $n .49$ - Il boomerang delle politiche che favoriscono la rendita, "Agriregionieuropa" dicembre.

Strambi G. (2017), La questione delle terre incolte e abbandonate e le leggi sulle "banche della terra", "Rivista di diritto agrario" n. 1.

Trapè I. (2016), Small farming e cambiamenti climatici, “Agricoltura Istituzioni Mercati” n. 2.

Victoria M.A. (2020), Nuevos derechos humanos de los campesinos en el marco de la Declaración de Naciones Unidas de 2018, "Revista Iberoamericana de Derecho Agrario" marzo. 


\title{
PEASANT AGRICULTURE BETWEEN LOCAL AND GLOBAL CONSIDERATIONS
}

\author{
Summary
}

This article deals with the issue of so-called peasant agriculture. It will first analyse the draft law "on the protection and valorisation of peasant agriculture" currently under discussion in the Italian Parliament, in particular the definition of the terms "azienda agricola Contadina" (peasant farm) and "agricoltore contadino" (peasant-farmer), while highlighting the arguments put forward by the citizens' movement that supports the project, and taking into account the international context in which these arguments had matured. In relation to this last aspect, elements of the UN Declaration on the rights of peasants and other people working in rural areas who will be included in the Italian legal system thanks to the law under discussion, will be analysed. The debate around peasant agriculture offers interesting insights not only on the role that non-industrialised and small-scale agriculture plays in achieving objectives of global importance such as food security or the fight against climate change and, more generally, how the challenge of achieving sustainable development posed by the UN with Agenda 2030 is relevant also from the point of view of traditional institutions of Italian agricultural law, such as the "imprenditore agricolo" (agricultural entrepreneur) or "coltivatore diretto" (direct farmer).

Keywords: peasant agriculture, rights of peasants, food security, food sovereignty, agroecology

\section{L'AGRICOLTURA CONTADINA FRA ISTANZE LOCALI E GLOBALI}

\section{Riassunto}

Il presente contributo ha per oggetto l'agricoltura cosiddetta contadina. Partendo dall'esame della proposta di legge "per la tutela e la valorizzazione dell'agricoltura contadina", attualmente in discussione al Parlamento italiano, e in particolare della definizione di "azienda agricola contadina" e "agricoltore contadino", si mettono in evidenza le istanze del movimento civile che sostiene il disegno di legge e il contesto internazionale in cui esse si sono sviluppate. In relazione a quest'ultimo aspetto, verranno esaminati gli elementi della Dichiarazione dell'ONU sui diritti dei contadini e delle altre persone che lavorano nelle aree rurali che verrebbero implementati nell'ordinamento italiano grazie al disegno di legge italiano. Il dibattito intorno all'agricoltura contadina offre, infatti, interessanti spunti di riflessione sul ruolo dell'agricoltura non industrializzata e di piccole dimensioni nel perseguire obiettivi di interesse globale come la food security e la lotta al cambiamento climatico e, più in generale, la sfida dello sviluppo sostenibile posta dall'ONU con l'Agenda 2030, ma anche sugli istituti tradizionali del diritto agrario italiano, quali quello dell'imprenditore agricolo e del coltivatore diretto.

Parole chiave: agricoltura contadina, diritti dei contadini, sicurezza alimentare, sovranità alimentare, agroecologia 